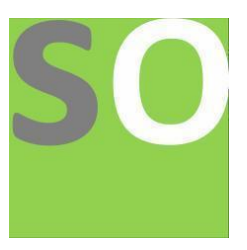

Article title: Mpemba Effect- the Effect of Time

Authors: Jianan Wang[1]

Affiliations: College of Physics and Energy, Shenzhen University, Shenzhen 518060, Guangdong, P. R. China[1]

Orcid ids: 0000-0001-7953-2808[1]

Contact e-mail: topsaving@163.com

License information: This work has been published open access under Creative Commons Attribution License http://creativecommons.org/licenses/by/4.0/, which permits unrestricted use, distribution, and reproduction in any medium, provided the original work is properly cited. Conditions, terms of use and publishing policy can be found at https://www.scienceopen.com/.

Preprint statement: This article is a preprint and has not been peer-reviewed, under consideration and submitted to ScienceOpen Preprints for open peer review.

DOI: 10.14293/S2199-1006.1.SOR-.PPXTYIJ.v2

Preprint first posted online: 18 August 2021

Keywords: Mpemba effect, inverse Mpamba effect, nature of time , time 


\title{
Mpemba Effect- the Effect of Time
}

\author{
Jian'an Wang \\ Department of Physics, Shenzhen University, Shenzhen, China \\ Email: wja@szu.edu.cn
}

\section{Abstract:}

This paper draws the following conclusions on the nature of time by analyzing the relationship between time and speed, the relationship between time and gravitational field, the gravitational redshift of the photon, and the black-body radiation theorem:

1. Time on an object is proportional to the amount of energy flowing out (or in) per unit time (observer's time) per unit surface area of the object.

2. When an object radiates energy outward:

$$
t^{\prime}=\mu B(\mathrm{~T})=\mu \sigma T^{4}=\mu \frac{n h v}{s t}
$$

Where $t^{\prime}$ is the time on the object, $\mu$ is a constant, $B(T)$ is the radiosity, the total energy radiated from the unit surface area of the object in unit time (observer's time), $\sigma$ is the Stefan-Boltzmann constant, $\mathrm{T}$ is the absolute temperature, $\mathrm{n}$ is the number of the photons radiated, $v$ is the average frequency of the photons radiated, $s$ is the surface area of the object and $t$ is the time on the observer.

3. When the object radiates energy outward, the higher the energy density of the space (for example the stronger the gravitational field of the space), the smaller the radiosity $B(T)$ of the object in the space, the longer the average wavelength of the light quantum emitted by the object, the slower the time on the object, the longer the life of the system.

4. When the object radiates energy outward, the faster the object moves relative to the ether, the higher the energy density of the local space in which the object is located, the smaller the radiosity $B(T)$ of the object, the longer the average wavelength of the light quantum radiated by the object, the slower the time on the object, and the longer the life of the system.

5. When the object radiates energy outward, the higher the temperature of the object, the greater the object's radiosity $\mathrm{B}(\mathrm{T})$, the shorter the average wavelength of the light quantum radiated by the object, the faster the time on the object, and the shorter the life of the system.

Applying the above conclusions about the nature of time, the author analyzes the Mpemba effect and the inverse Mpemba effect, and reaches the following conclusion: the Mpemba effect is the time effect produced when heat flows from objects into space, and the "inverse" Mpemba effect is the time effect produced when heat flows from space into objects.

\section{Key words:}

Mpemba effect, inverse Mpemba effect, nature of time, time, time dilation, gravitational redshift, blackbody radiation 


\section{Introduction:}

" Mpemba effect ", also known as" Mupainmubar effect ", refers to the phenomenon that a slightly hotter liquid freezes first than a slightly cooler one under the same volume, mass and cooling conditions. In 1963, at a high school in Tanzania, there was a junior three student named Mpemba who enjoyed making ice cream with his classmates. They add sugar to boiling fresh milk, let it cool, pour it into a latticed container, and place it in the freezer. One day, Mpemba realized that the freezer room was running short of space. Fearing that the other students would be the first to use it, he quickly added sugar to the boiling milk and sent it to the refrigerator before it cooled down. An hour and a half later, Mpemba noticed something that puzzled him: hot milk freezes faster than cold milk. What's going on here? Confused Mpemba hurriedly ran to ask the teacher, but unfortunately, from junior high school to high school, no teacher took his problem seriously, and some people thought he was absurd and said he was lying. Until one day, Dr. Osborn, the head of the physics department of the University of Dar es Salaam, visited his school, and he seized the opportunity to consult Dr. Osborn. As a result, the doctor not only did not scoff at it, but also took him back to the laboratory to do the experiment. In 1969, Mpemba and Dr. Osborne wrote a paper on this effect and named it "Mpemba effect ".

In fact, hot water freezes faster than cold water is a mystery that has been around for thousands of years. Historically, Aristotle, Francis Bacon and Descartes have described the phenomenon in different ways, but none of them has attracted much attention [1]

More than 50 years after the "Mpemba effect " was officially named, scientists have done many experiments and written many papers want to prove the principle behind the phenomenon[2],[3],[4],[5],[6],[7] [8], but there is still no conclusion.

Recently, two physicists Avinash Kumar and John Bechhoefer from simon fraser university, Canada, have bypassed the complexity of water by replacing water molecules with tiny glass beads, developing a method to exhibit the Mpemba effect in a controllable environment, confirming that when two systems with different initial temperatures are cooled to the same temperature, the system with higher initial temperatures can take less time than the system with lower temperatures.[9]

The result suggests that the Mpemba effect is not only present in glass beads and water, but is more likely to be present in nature in general.

\section{Analyzes the nature of time by the relation between time and velocity, time and gravitational field, gravitational redshift, and the black-body radiation theorem}

The relationship between time and speed is revealed, that is, the faster the speed, the slower the time, and the relationship between time and gravitational field is revealed also, that is, the stronger the gravitational field, the slower the time. The author believes that the above two reflect the same property of time: "The higher the energy density of space, the slower the time" [10]. Because physical space is composed of ether (energy), the faster the object moves relative to ether, the more ether (energy) the object gathers, the greater the kinetic energy of the object, the higher the energy density of the local space where the object is located, so the time is slower [10]. According to formula (5.17) [10]: 


$$
\mathrm{t}=\mathrm{t}_{0} \sqrt{1-\frac{\mathrm{v}^{2}}{\mathrm{c}^{2}}}
$$

where, $t_{0}$ is the time when the object is at rest relative to the ether, i.e., $v=0$; $t$ is the time when the object is moving with a speed $v$ relative to the ether, and formula (5.12) [10]:

$$
\mathrm{m}=\frac{\mathrm{m}_{0}}{\sqrt{1-\frac{\mathrm{v}^{2}}{\mathrm{c}^{2}}}}
$$

and the formula:

$$
\mathrm{mc}^{2}=\frac{\mathrm{m}_{0} \mathrm{c}^{2}}{\sqrt{1-\frac{\mathrm{v}^{2}}{\mathrm{c}^{2}}}}=\mathrm{m}_{0} \mathrm{c}^{2}+\mathrm{m}_{0} \mathrm{c}^{2}\left\{\frac{1}{2}\left(\frac{\mathrm{v}^{2}}{\mathrm{c}^{2}}\right)+\frac{3}{8}\left(\frac{\mathrm{v}^{2}}{\mathrm{c}^{2}}\right)^{2}+\cdots\right\}=\mathrm{E}_{0}+\mathrm{E}_{\mathrm{k}}
$$

where $m$ is the moving mass of the object, $m_{0}$ is the static mass of the object, $c$ is the speed of light in vacuum, $E_{k}$ is the kinetic energy of the object, and $E_{0}$ is the static energy of the object, we can get:

$$
\mathrm{t}=\left(\frac{\mathrm{E}_{0}}{\mathrm{E}_{0}+\mathrm{E}_{\mathrm{k}}}\right) \mathrm{t}_{0}
$$

Divide the numerator and denominator on the right side of equation (4) by the volume of the object, and we can get:

$$
\mathrm{t}=\left(\frac{\rho_{0}}{\rho_{0}+\rho}\right) \mathrm{t}_{0}=\frac{\mathrm{t}_{0}}{\left(1+\frac{\rho}{\rho_{0}}\right)}
$$

In the formula, $\rho$ is the spatial energy density caused by the kinetic energy of the object, and $\rho_{0}$ is the energy density of the object itself. According to formula (4) and (5), the greater the kinetic energy, the greater the space energy density, the slower the time. Therefore, the acceleration of the sun's motion speed in the Galaxy would slow down the time on the sun, and thus reduce the solar radiation power and lead to the generation of the Ice Age [11].

Because the stronger the gravitational field, the higher the space energy density, the stronger the gravitational field, the slower the time, that is, the higher the space energy density, the slower the time.

Because the energy density of any field $\rho$ and the field strength $E$ satisfy the relation:

$$
\rho=\mathrm{eE}^{2}
$$

In the above equation, e is the coefficient. According to equations (5) and (6), we can get:

$$
\mathrm{t}=\frac{\mathrm{t}_{0}}{\left(1+\frac{\mathrm{eE}^{2}}{\rho_{0}}\right)}
$$

In the above equation, $\mathrm{t}$ is the time on the object when the object is in the field $\mathrm{E}$, and $\mathrm{t}_{0}$ is the time on the object when the field is zero. Obviously, the stronger the field $E$ is, the slower the time $t$ is.

In formula (7), E can be the gravitational field, electrostatic field, magnetic field or other fields. So we can predict that the stronger the electrostatic field or magnetic field, the slower the time on the object in the electrostatic field or magnetic field.

We can prove that, from formula (7), we can get the following relation between time and gravitational field in general relativity: 


$$
t=t_{0} \sqrt{1-\frac{2 G M}{c^{2} r}}
$$

In the above equation, $\mathrm{t}$ is the time interval in the gravitational field of the planet, $\mathrm{t}_{0}$ is the time interval outside the gravitational field of the planet, $M$ is the mass of the planet, $r$ is the radius of the planet, $G$ is the gravitational constant, and $c$ is the speed of light in a vacuum. From formula (7) we can get:

$$
t=t_{0} \sqrt{1-\frac{2 \mathrm{eE}^{2}}{\rho_{0}}}
$$

Since the specific gravity of a general object on the Earth is basically the same as that of the Earth, in Formula (9) we can approximately replace the energy density of a general object on the Earth with the energy density of the Earth:

$$
\rho_{0}=\frac{M c^{2}}{\frac{4 \pi}{3} r^{3}}
$$

where $M$ is the mass of the earth, $c$ is the speed of light in a vacuum, and $r$ is the radius of the earth. Substituting formula (10) and the following formula for the strength of the earth's gravitational field:

$$
E=G \frac{M}{r^{2}}
$$

into Formula (9), we get:

$$
t=t_{0} \sqrt{1-\frac{8 \pi e G^{2}}{3} \frac{M}{c^{2} r}}
$$

In formula (12), let

$$
\mathrm{e}=\frac{3}{4 \pi \mathrm{G}}
$$

then formula (8) can be obtained.

From the gravitational red shift of the photon, the stronger the gravitational field is, the longer the wavelength of the photon is, and the smaller the energy of the photon is. Because the stronger the gravitational field is, the slower the time is. Therefore, the stronger the gravitational field is (the higher the energy density of space), the less the energy of the light quantum radiated by the object in the gravitational field is, and the slower the time on the object is. So, we can infer that the shorter the average wavelength of the photons emitted by the object, the faster the time on the object.

Figure 1 shows the relation between the emissionvity $I(\lambda, T)$ of the blackbody at different temperatures $T$ (that is, the energy radiated from unit area and unit solid Angle at unit wavelength intervals in unit observer time) and wavelength $\lambda$ in accordance with Planck blackbody radiation theorem. 


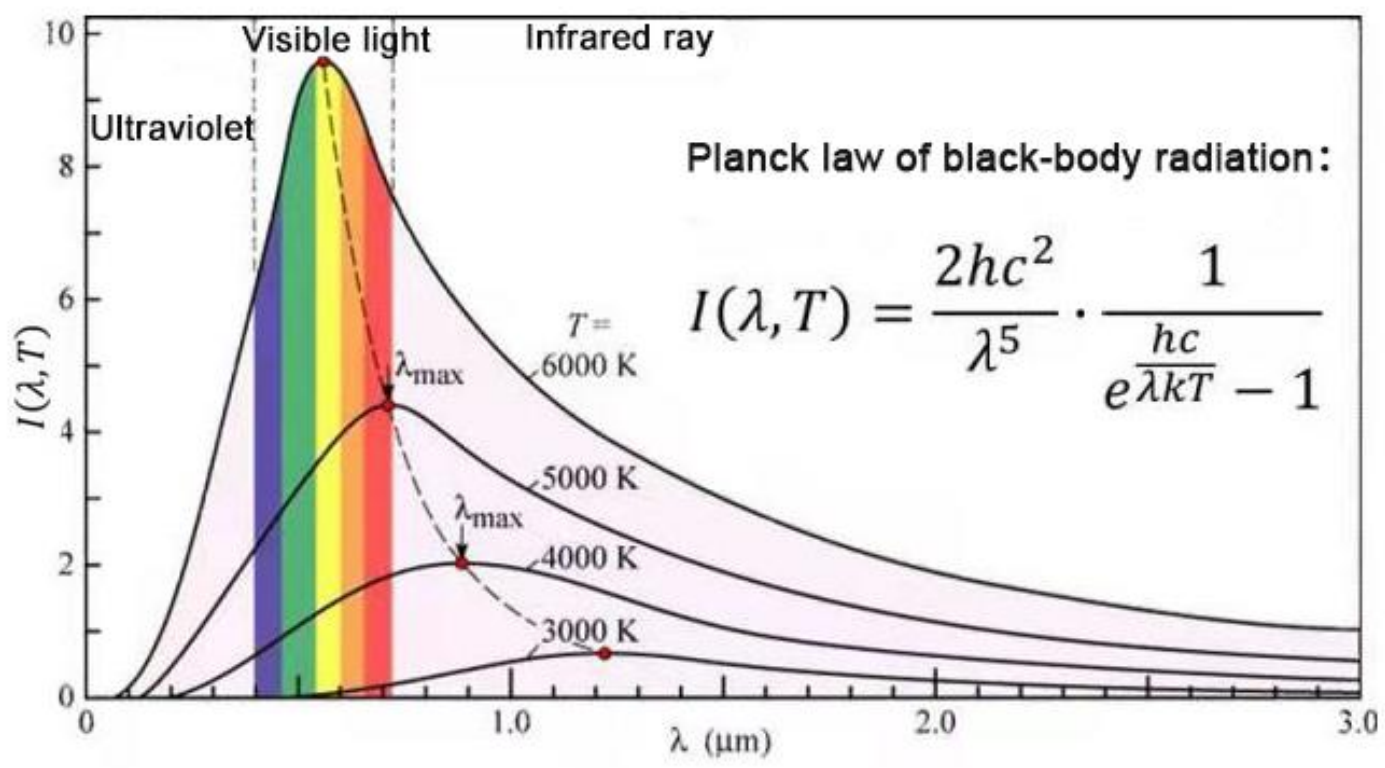

Figure 1

As can be seen from the figure above, the higher the temperature of the black body, the shorter the wavelength of its maximum emissivity $\lambda_{m}$, and the higher the emissivity $I(\lambda, T)$ corresponding to the same wavelength. That is, the higher the temperature, the shorter the wavelength of the photons emitted by the blackbody, and the greater the emissivity. Because any object can be approximated as a black body, so we can speculate: when the space energy density is certain, the higher the temperature of the object, the faster the time on the object. Therefore, increasing the temperature of an object has the same effect as reducing the energy density of space (such as reducing the intensity of the gravitational field). Both can increase the frequency of radiation photons and speed up the time on the object.

Because all changes in an object (including the changes in position) are essentially the energy exchange between the object and the space, time is essentially the rate of energy exchange between object and space.

According to Stefan-Boltzmann's law, the total energy radiated from a unit surface area of a black body with absolute temperature $T$ in unit time (observer time) is

$$
B(T)=\sigma T^{4}
$$

Where $\sigma$ is the Stefan-Boltzmann constant (also known as the blackbody radiation constant), equal to $5.67 \times 10^{-8} \mathrm{~W} /\left(\mathrm{m}^{2} \cdot \mathrm{K}^{4}\right)$, and $\mathrm{T}$ is the temperature of the blackbody. Because the higher the temperature of the blackbody, the greater the radiosity $\mathrm{B}(\mathrm{T})$ of the blackbody, the author conjecture that the time on the blackbody is proportional to the radiosity of the blackbody, that is, to the energy radiated from the unit surface area of the blackbody in the unit observer time:

$$
t^{\prime} \propto B(\mathrm{~T})=\sigma T^{4}=\frac{n h v}{s t}
$$

Where $t^{\prime}$ is the time on the black body, $n$ is the number of the photons radiated, $v$ is the average frequency of the photons radiated, $s$ is the surface area of the black body, $t$ is the observer time. 
For two black bodies at temperatures $\mathrm{T} 1$ and $\mathrm{T} 2$, we have:

$$
\mathrm{t}_{1}^{\prime} \propto \mathrm{B}\left(\mathrm{T}_{1}\right)=\sigma \mathrm{T}_{1}^{4}=\frac{\mathrm{nh} v_{1}}{\mathrm{st}}
$$

and

$$
\mathrm{t}_{2}^{\prime} \propto \mathrm{B}\left(\mathrm{T}_{2}\right)=\sigma \mathrm{T}_{2}^{4}=\frac{\mathrm{nhv_{2 }}}{\mathrm{st}}
$$

From equations (16) and (17), it can be obtained:

$$
\frac{\mathrm{t}_{1}^{\prime}}{\mathrm{t}_{2}^{\prime}}=\frac{\mathrm{T}_{1}^{4}}{\mathrm{~T}_{2}^{4}}
$$

If $\mathrm{T}_{1}=343 \mathrm{~K}$ and $\mathrm{T}_{2}=263 \mathrm{~K}$, then we have:

If $\mathrm{T}_{1}=10000 \mathrm{~K}, \mathrm{~T}_{2}=1000 \mathrm{~K}$, then:

$$
\frac{\mathrm{t}_{1}^{\prime}}{\mathrm{t}_{2}^{\prime}}=\frac{\mathrm{T}_{1}^{4}}{\mathrm{~T}_{2}^{4}}=\left(\frac{343}{263}\right)^{4}=2.891
$$

$$
\frac{\mathrm{t}_{1}^{\prime}}{\mathrm{t}_{2}^{\prime}}=\frac{\mathrm{T}_{1}^{4}}{\mathrm{~T}_{2}^{4}}=\left(\frac{10000}{1000}\right)^{4}=10000
$$

From the above analysis, we can conclude that the higher the temperature of the blackbody, the greater the average energy of the photons radiated by the blackbody, the faster the time passes on the blackbody, and the shorter the life of the system.

Although the blackbody is only an ideal object, any object can be approximated as a blackbody, and its thermal radiation will follow Planck's law. Because any system (such as the heat of an object, or the deuterium tritium nucleofusion energy of a star) is composed of quantum. Based on the above analysis of the relationship between time and velocity, the relationship between time and gravitational field, gravitational redshift and blackbody radiation theorem, we can draw the following conclusions:

1. Time on an object is proportional to the amount of energy flowing out (or in) per unit time (observer time) per unit surface area of the object.

2. When an object radiates energy outward:

$$
\mathrm{t}^{\prime}=\mu \mathrm{B}(\mathrm{T})=\mu \sigma \mathrm{T}^{4}=\mu \frac{\mathrm{nh} v}{\mathrm{st}}
$$

where $t^{\prime}$ is the time on the object, $\mu$ is a constant, $B(T)$ is the radiosity, the total energy radiated from the unit surface area of the object in unit time (observer's time), $\sigma$ is the StefanBoltzmann constant, $\mathrm{T}$ is the absolute temperature, $\mathrm{n}$ is the number of the photons radiated, $v$ is the average frequency of the photons radiated, $s$ is the surface area of the object and $t$ is the time on the observer.

3. When an object radiates energy outward, the higher the energy density of the space (for example the stronger the gravitational field of the space), the smaller the radiosity $\mathrm{B}(\mathrm{T})$ of the object in the space, the longer the average wavelength of the light quantum emitted by the object, the slower the time on the object, the longer the life of the system.

4. When an object radiates energy outward, the faster the object moves relative to the ether, the higher the energy density of the local space in which the object is located, the smaller the radiosity $B(T)$ of the object, the longer the average wavelength of the light quantum radiated by the object, the slower the time on the object, and the longer the life of the system.

5. When an object radiates energy outward, the higher the temperature of the object, the greater the object's radiosity $\mathrm{B}(\mathrm{T})$, the shorter the average wavelength of the light quantum 
radiated by the object, the faster the time on the object, and the shorter the life of the system. Based on the above time-related conclusions, we can explain why quasars have such short lifetimes [12] [13],[14],[15]. Due to the extremely high temperature of quasars (up to $4 \times 10^{13} \mathrm{~K}$ ), the average wavelength of the photons emitted by the quasars is very short, and the radiosity $B(T)$ of the quasars is very high, which means that the time on the quasars passes very quickly, so the lifetime of the quasars is very short.

In the same way, we can explain why the greater the stellar mass, the shorter the lifetime. Because the more massive the star, the hotter the star. The hotter the star, the greater the star's radiosity $\mathrm{B}(\mathrm{T})$, that is, the faster the time on the star. So, the more massive the star, the shorter the lifetime of the star.

It can be seen from conclusion 4 above about time, the faster the sun moves relative to the Galaxy, the slower the time on the sun, the longer the average wavelength of the photons emitted by the sun, the smaller the radiation power of the sun, and the fewer sunspots. That is also why the Earth enters the ice age at regular intervals. Because the sun's speed around the galaxy is not uniform, when the sun moves in the orbital segments of greater speed, the solar radiation power will be reduced, so the earth will receive less sunlight, the temperature on the Earth will drop. In the orbital segment that is closest to the Galactic center, the Sun moves at the highest speed relative to the Galaxy, so once the solar system enters this orbital segment of the Galaxy, the earth enters the Great Ice Age.

The author believes that the Mpemba effect and the effect observed by Avinash Kumar and John Bechhoefer in the experiment [9] are also the time effect.

\section{The explanation of the Mpemba effect}

As we can see from the conclusions in the above section, when the energy density of space remains constant, the higher the temperature of the object, the greater the radiosity $\mathrm{B}(\mathrm{T})$ of the object, the shorter the average wavelength of the photons radiated by the object, the faster the time on the object, and the shorter the life of the system. Therefore, although a high temperature object needs to emit more heat from the beginning to the end of the temperature than a same object of lower temperature, the average temperature of the high temperature object in the process of cooling from the beginning to the end of the temperature is higher than that of the low temperature object, the average time on the high temperature object is faster than that on the low temperature object, which makes it possible for the high temperature object to reach the end of the temperature first. In the Mpemba experiment, the water is not in a vacuum, but in the air, so in addition to the presence of heat radiation in water, there are also heat exchange and heat convection between water and air. Nevertheless, the time in the cup of water is still proportional to the energy inflow (or outflow) per unit surface area in unit observer time.

In the Mpemba experiment, because the refrigerator pumps away the heat of the space in time to maintain the same temperature of the space in which the two cups of water are located, the difference of time between the two cups of water are determined only by the temperature difference between the two cups of water. If the initial temperature of the cold water and the temperature difference between the two cups of water are set properly, then it should be observed that the hot water freezes first.

Since the substance (water) involved in the Mpemba experiment does not produce any $7 / 9$ 
nuclear reactions, the static mass of the substance (water) is independent of the system under study, which is simply composed of the heat contained in the substance(water). Therefore, the change of time on the system studied does not require much energy (heat) flowing from the object (the water in the cup).

\section{Why is the Mpemba efect difficult to observe?}

Many people did the Mpemba experiment and most people observed that the lower temperature water froze first. Why? The author believes that the main reasons are:

1. The power of the refrigerator is too small relative to the amount of water put in, so the temperature of the space around the high temperature water is much higher than that around the low temperature water. As a result, the time difference between the high temperature water and the low temperature water is reduced, and the low temperature water freezes first. This is why we observe that a basin of warm water above room temperature turns to roomtemperature cold water more quickly than a same basin of boiling water o

2. The initial temperature of low temperature water and the initial temperature difference between high temperature water and low temperature water are not set properly. Because the loss of heat and the passage of time in the Mpemba experiment are carried out simultaneously and affect each other, whether hot water or cold water freezes first is related to the initial temperature of low temperature water and the initial temperature difference between high temperature water and low temperature water.

The explanation of the "inverse" Mpemba effect.

The "inverse" Mpemba effect is the effect in which "heating a cooled, far-from-equilibrium object takes less time than another object that is initially closer to equilibrium under the same volume, mass and heating conditions." [16]. In this case, the time on the object is proportional to the amount of energy flowing into the object per unit surface area, per unit time (observer time). So, when the ambient temperature stays the same, the energy flows into the cold object faster, and the time on the cold object is faster. Therefore, although the low temperature object needs to absorb more heat from the temperature start point to the end point (equilibrium) than the high temperature object, since the average temperature of the low temperature object is lower than that of the high temperature object in the process of heating from the starting point to the end point of temperature, the average speed of time passing on the low temperature object is greater than that on the high temperature object, thus the low temperature object reach the end point(equilibrium) of temperature first.

\section{Conclusions:}

1. Time on an object is proportional to the amount of energy flowing out (or in) per unit time (observer time) per unit surface area of the object.

2. In the Mpemba experiment the system under study is composed of the heat contained in the 
object. The Mpemba effect is the time effect produced when heat flows from objects into space. The "inverse" Mpemba effect is the time effect produced when heat flows from space into objects.

\section{References:}

[1] Dorsey, N. Ernest (1948). "The freezing of supercooled water". Trans. Am. Phil. Soc. American Philosophical Society. 38 (3): 247-326. doi:10.2307/1005602. JSTOR 1005602.

[2] Ball, Philip (April 2006). Does hot water freeze first?. Physics World, pp. 19-26.

[3] How to Fossilize Your Hamster: And Other Amazing Experiments for the Armchair Scientist, ISBN 1-84668-044-1

[4] Bregović, Nikola; Mpemba effect from a viewpoint of an experimental physical chemist, 2013

[5] Jin, Jaehyeok; Goddard III, William A. (2015). "Mechanisms Underlying the Mpemba Effect in Water from Molecular Dynamics Simulations". Journal of Physical Chemistry C. 119 (5): 26222629. doi:10.1021/jp511752n

[6] Tao, Yunwen; Zou, Wenli; Jia, Junteng; Li, Wei; Cremer, Dieter (2017). "Different Ways of Hydrogen Bonding in Water - Why Does Warm Water Freeze Faster than Cold Water?". Journal of Chemical Theory and Computation. 13: 55. doi:10.1021/acs.jctc.6b00735.

[7] Lu, Zhiyue; Raz, Oren (2017-05-16). "Nonequilibrium thermodynamics of the Markovian Mpemba effect and its inverse". Proceedings of the National Academy of Sciences. 114 (20): 5083-5088. arXiv:1609.05271 Freely accessible.

[8] Lasanta, Antonio; Vega Reyes, Francisco; Prados, Antonio; Santos, Andrés (2017). "When the Hotter Cools More Quickly: Mpemba Effect in Granular Fluids". Physical Review Letters. 119 (14): 148001. arXiv:1611.04948 Freely accessible.

[9] Kumar, A., Bechhoefer, J. Exponentially faster cooling in a colloidal system. Nature 584, 64-68 (2020). https://doi.org/10.1038/s41586-020-2560-x

[10] Wang, J.A. (2019) Journal of Modern Physics, 10, 1615-1644.

https://doi.org/10.4236/jmp.2019.1014107

[11] Wang, J.A. (2020) Journal of Modern Physics, 11, 407-431. https://doi.org/10.4236/jmp.2020.113026

[12] Zuo W W, Wu X B. The mystery of quasar power (in Chinese). Chin Sci Bull, 2016, 61: 11571163, doi: 10.1360/N972016-00108

[13] A dozen quasars in the early universe appear to have shut down in just a few years, baffling astronomers. By Shannon Hall on November 23, 2015. http://www.scientificamerican.com/article/the-case-of-the-disappearing-quasars/

[14] THE DISCOVERY OF THE FIRST "CHANGING LOOK" QUASAR: NEW INSIGHTS INTO THE PHYSICS AND PHENOMENOLOGY OF ACTIVE GALACTIC NUCLEI Stephanie M. LaMassa et al. 2015 The Astrophysical Journal, Volume 800 , Number 2

[15] Toward an Understanding of Changing-look Quasars: An Archival Spectroscopic Search in SDSS John J. Ruan et al. 2016 The Astrophysical Journal 826188

[16] Lu Zhiyue,Raz Oren. Nonequilibrium thermodynamics of the Markovian Mpemba effect and its inverse.[J]. Proceedings of the National Academy of Sciences of the United States of America,2017,114(20). 